\title{
Prehabilitation in elderly patients scheduled for liver resection and protocol for Recovery Of Surgery in Elderly
}

\author{
Ramkumar Mohan ${ }^{1}$, Cheong Wei Terence Huey², Sameer Junnarkar², Jee Keem Low ${ }^{2}$, Vishal G. Shelat ${ }^{2}$ \\ ${ }^{1}$ Yong Loo Lin School of Medicine, National University of Singapore, Singapore. \\ ${ }^{2}$ Senior Consultant, Hepatopancreaticobiliary Surgery, Tan Tock Seng Hospital, Singapore.
}

Correspondence to: Ramkumar Mohan, Yong Loo Lin School of Medicine, National University of Singapore, Singapore. E-mail:m.ramkumar@u.nus.edu

How to cite this article: Mohan R, Huey CWT, Junnarkar S, Low JK, Shelat VG. Prehabilitation in elderly patients scheduled for liver resection and protocol for recovery of surgery in elderly. Hepatoma Res 2020;6:13. http://dx.doi.org/10.20517/2394-5079.2019.53

Received: 25 Dec 2019 First Decision: 2 Mar 2020 Revised: 12 Mar 2020 Accepted: 18 Mar 2020 Published: 26 Mar 2020

Science Editor: Bruno Nardo Copy Editor: Jing-Wen Zhang Production Editor: Jing Yu

\begin{abstract}
Ageing population of first world economies pose unique challenges to surgical community. Enhanced recovery after surgery protocols and pathways do not attempt to optimize or enhance physical function of patients by customized program of physical activity. Increasingly, prehabilitation programs (PP) have gained momentum in orthopaedics, urology, colorectal surgery and hepatopancreaticobiliary surgery. Current evidence of PP in various elective surgical procedures have shown improved outcomes with minimal to none drawback or harm. There is emerging evidence of role of PP in elective liver resection. The aim of this paper is to review the basis of PP and share local multidisciplinary team protocol specifically customized to frail and elderly population - Recovery Of Surgery in Elderly.
\end{abstract}

Keywords: Prehabilitation, liver resection, hepatocellular carcinoma, pre-operative exercise, ageing

\section{INTRODUCTION}

Liver is the largest solid intra-abdominal organ and common site for primary and metastatic cancers. The most common liver malignancy is secondary from gastrointestinal tract with colorectal liver metastasis being the commonest. Hepatocellular carcinoma is the most common primary liver cancer and fifth most common cancer worldwide ${ }^{[1]}$. Hepatocellular carcinoma occurs in elderly population and the incidence is expected to rise with epidemic of diabesity as well as ageing population. With increased participation in

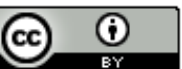

(C) The Author(s) 2020. Open Access This article is licensed under a Creative Commons Attribution 4.0 International License (https://creativecommons.org/licenses/by/4.0/), which permits unrestricted use sharing, adaptation, distribution and reproduction in any medium or format, for any purpose, even commercially, as long as you give appropriate credit to the original author(s) and the source, provide a link to the Creative Commons license, and indicate if changes were made.

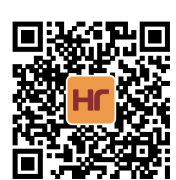


screening programs for Hepatitis B, increasing awareness and advances in imaging technology facilitating early diagnosis, surgery has an increasing role in management of hepatocellular carcinoma. Age-related comorbidities increase operative morbidity and impacts peri-operative outcomes. Enhanced recovery after surgery (ERAS) programs emphasize on intra-operative and post-operative care standardization with minimal guidance on pre-operative phase except nutritional optimization and carbohydrate loading. Pre-operative phase provides an opportunity for surgeons to optimize patients to meet up for impending physiologic stress imposed by surgery. In this paper, we discuss the role of prehabilitation program in elderly patients scheduled for liver resection and also discuss the current protocol of Recovery Of Surgery in Elderly (ROSE) program.

\section{AGEING}

Along with greying of hair, development of cataracts, degenerative bone disorders and declining cognition, ageing leads to a myriad of other physiologic changes in organ systems. Atherosclerosis, hypertension and decreased cardiac output are noted in the cardiovascular systems. Impaired gas exchange, reduction in vital capacity and reduced expiratory flow rates are noted in pulmonary system. Furthermore, decline in lean body mass, createnine clearance reduction, hepatic drug metabolism impairment and gastrointestinal motility reduction are noted. This functional and metabolic alteration is compounded with polypharmacy for managing chronic illnesses. Figure 1 shows age related physiologic changes in organ systems. Frailty and sarcopenia are common in elderly too ${ }^{[2]}$.

\section{FRAILTY AND SARCOPENIA}

Frailty is a pre-disability syndrome in the elderly when exposed to stressors with increased risk of disability or need for hospitalization. The European Working Group on Sarcopenia in Older People has defined sarcopenia as progressive, generalized loss of skeletal mass with associated functional losses ${ }^{[3]}$. Low skeletal muscle mass ${ }^{[4]}$ and low functional capacity ${ }^{[5]}$ associated with sarcopenia lead to increased post-operative complications as well as reduced long term survival. As such the elderly are vulnerable, especially when faced with peri-operative stress. Morley et al. ${ }^{[6]}$, reported that sarcopenia and frailty are closely linked to ageing. They reported decreased hand grip strength, walking speed and weight in the elderly and concluded lack of muscle usage as one of the reasons which impacts outcomes. Prehabilitation aims to mitigate the drawbacks of frailty.

\section{PREHABILITATION}

Rehabilitation is integrated in routine clinical medicine. It focuses on recovery following a surgical stressor. Prehabilitation is defined as the process of augmenting functional capacity before surgery with aim of reducing post-operative morbidity and/or mortality. This is done through a personalized regimen of aerobic, functional and strength training. It aims to optimize pre-operative functional cardiorespiratory and nutritional reserves. As the scope of prehabilitation overlaps with concept of rehabilitation and rehabilitation is integral to orthopaedic surgery, orthopaedic teams were early adopters of prehabilitation ${ }^{[7]}$. Rooks et al. ${ }^{[8]}$ reported 108 patients scheduled for total hip arthroplasty and total knee arthroplasty and showed improvements in preoperative and post-operative muscle strength and reduced need for inpatient rehabilitation. In a systemic review by Coudeyre et al. ${ }^{[7]}$, prehabilitation contributed to reduced hospitalization and improved discharge conditions in patients with total hip arthroplasty and total knee arthroplasty. A pilot randomized study including 30 elderly patients above 65 years old undergoing total hip arthroplasty showed that home based physical therapy was feasible to implement and it improved 6-min walk test ${ }^{[9]}$ as compared to standard care. Prehabilitation is also implemented in urology and colorectal surgery. Au et al. ${ }^{[10]}$ reported increased inpatient physical activity on post-operative day 1 in patients undergoing radical prostatectomy. A randomized controlled trial by Painter et al. ${ }^{[11]}$ included 167 patients 


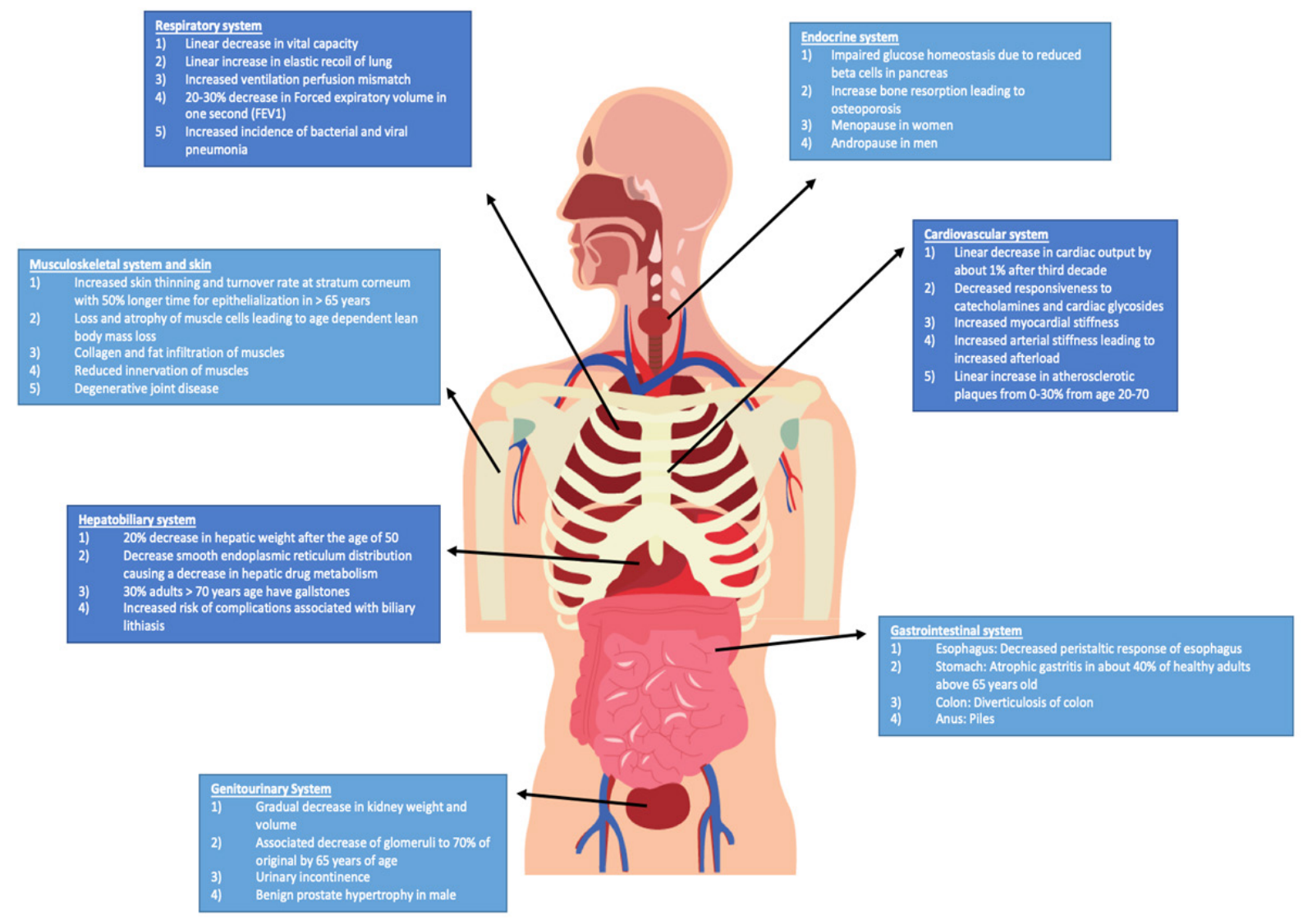

Figure 1. Age related physiological changes by system ${ }^{[2]}$

undergoing renal transplant and showed improved graft function, aerobic fitness, quality of life and patient reported physical functionality. Similar reports were also found in reviews of patients undergoing renal transplant ${ }^{[12,13]}$. Mayo et al. ${ }^{[14]}$ reported 95 patients with colorectal resections and showed increased functional outcomes such as in the 6-min walking test in $33 \%$ of patients. A recent meta-analysis by Gillis et al. ${ }^{[15]}$ also showed that prehabilitation could reduce post-operative admissions by two days in patients who underwent colorectal surgery. A systemic review by Cabilan et al. ${ }^{[16]}$, included 13 orthopedic, one colorectal, two cardiac and one foregut study. Prehabilitation did not demonstrate benefits in objective and self-reported function or reduction in inpatient rehabilitation admission. Some studies include cognitive behavioral therapy in addition to prehabilitation and this introduces heterogeneity ${ }^{[17]}$. As such, more studies are needed to establish the role of prehabilitation and define patient selection criteria. Prehabilitation should be personalized based on various factors such as: (1) surgery: type, duration, expected blood loss, predicted morbidity and mortality; (2) patient factors: age, frailty, sarcopenia, co-morbidity, caregiver support; and (3) pathology: malignancy, burden of disease, malnutrition, cachexia, emotional impact.

\section{PREHABILITATION IN HEPATO-PANCREATICO-BILIARY SURGERY}

Hepato-pancreatico-biliary surgery is complex and many units have adopted prehabilitation. In a local study ${ }^{[18]}$ including 245 patients with liver resection the post-operative morbidity, 30-day mortality and 90-day mortality was $38.3 \%, 2.4 \%$ and $3.7 \%$ respectively. In a local study ${ }^{[19]}$ including 196 patients with pancreatic resection, the rate of grade $\mathrm{B}$ and $\mathrm{C}$ post-operative pancreatic fistula, 30-day mortality and 90-day mortality was $5.1 \%, 0.5 \%$ and $2 \%$, respectively. Prehabilitation has potential to improve peri-operative outcomes. In a propensity-score matched study including 76 patients undergoing major hepato-pancreatico-biliary 
surgery, Nakajima et al. ${ }^{[20]}$ reported improvements in serum albumin levels and reduction in hospital stay length but no difference in complication rates. Low serum albumin is associated with increased morbidity in elective and emergency procedures ${ }^{[21]}$. In 40 patients undergoing pancreaticoduodenectomy, Ausania et al. ${ }^{[22]}$ reported improvement in delayed gastric emptying but not in post-operative complications such as postoperative pancreatic fistula. Most studies do not report significant negative outcomes even though some trials fail to demonstrate benefit.

Outcomes of success of prehabilitation program are multifaceted: (1) mortality/morbidity related outcomes: disease progression during prehabilitation, infectious morbidity during prehabilitation; (2) resource related outcomes: total cost of care, value driven outcomes; and (3) efficacy related outcomes: length of stay, admission to rehabilitation unit, morbidity/mortality post operatively, long term disease progression, acceptance of chemotherapy and adjunct therapy post operatively.

Most trials report clinical outcomes through careful patient selection and elimination of emergent cases and those in need of urgent intervention. Janssen et al. ${ }^{[23]}$ has reported single centre uncontrolled before and after study including 627 patients aged 70 years and older and undergoing elective abdominal surgery for colorectal carcinoma or aortic aneurysm. The prehabilitation group received interventions to improve patients' physical health, nutritional status, frailty and anaemia prior to surgery. With a mean prehabilitation of 39 days duration, they showed that the incidence of delirium was reduced significantly from $11.7 \%$ to $8.2 \%(\mathrm{OR}=0.56,95 \% \mathrm{CI}$ : $0.32-0.98, P=0.043)$. Some studies such as that by Nielsen et al. ${ }^{[24]}$ report that even though intervention costs increase, overall costs reduce by about $15 \%$; however, this remains to be validated for liver resection.

Prehabilitation has also shown improved outcomes in patients with liver resection. For most of these patients the effects of ageing are compounded by ongoing cirrhosis and oncological burden. A prospective study including 104 patients treated with elective liver resection showed reduced overall complication rates by up to $22.9 \%$ and median length of hospital stay by 2.5 days less ${ }^{[25]}$. The authors also highlighted social benefits of prehabilitation. These included less social issues that may delay discharge, increased quality of life and reduced median cost by up to $16.5 \%{ }^{[25]}$. Though this study was not randomized and included small sample, it sets the precedent for positive outcomes of prehabilitation. Another trial by Dunne et al. ${ }^{[26]}$ on 35 randomized patients undergoing liver resection for colorectal liver metastases, reported significant preoperative score increase in both mental and physical aspects of the Short Form Health Survey 36. This supports the idea of both quality of life and physical fitness improvement due to prehabilitation. In a review by Tandon et al. ${ }^{[27]}$, elderly patients with cirrhosis have shown improvements in muscle mass, strength and functional capacity. They have also reported reduction in hepatic venous gradient and hepatic steatosis. These results were replicated in a review by Locklear et al. ${ }^{[28]}$ which showed such decreases in hepatic venous gradient and increased scores on 6-min walk tests for patients with end stage liver disease. Prehabilitation includes components of ERAS and more comprehensive preoperative strategy. ERAS has shown lower major complication rates and reduced cost in patients undergoing liver resection ${ }^{[29]}$. A systemic review on ERAS application in liver surgery by Brustia et al ${ }^{[30]}$ suggests positive outcomes such as reduced time taken for functional recovery by 2.5 days. Similar to ERAS, the American College of Surgeons have implemented a program called strong for surgery ${ }^{[31]}$, which is aimed at identifying and evaluating evidence-based practices to optimize the health of patients before surgery. It includes optimisation of nutrition, smoking cessation, pain control and prehabilitation. We have started ROSE program at Tan Tock Seng Hospital, Singapore to improve outcomes of elderly patients undergoing elective liver resection. In this paper we shall discuss our protocol of implementation of ROSE program.

\section{ROSE}

In Singapore, a population health strategy is designed to meet evolving healthcare needs for ageing society and "future ready". National Healthcare Group is the central cluster serving a population of 1.4 million 
and embraces the concept of "River of Life". This defines five segments of care - Living Well, Living with Illness, Crisis and Complex Care, Living with Frailty and Leaving Well - rooted by strong partnerships amongst care providers. Six population care streams feed into "River of Life" - Preventive Care, Primary Care, Hospital Care, Intermediate Care, Transitional and Community Care, End-of-Life and Long-Term Care. With the increasing burden of chronic diseases, primary care will take on an even bigger role in the community. Though there are reports of safe surgery with acceptable outcomes in elderly as compared to non-elderly ${ }^{[32,33]}$; elderly do have additional needs to ensure comparable outcomes. Hence, additional resources have to be invested to achieve good outcomes. ROSE program was initiated in 2018 by a multidisciplinary group of healthcare professionals who recognized the need for our elderly patients to be optimized prior to major abdominal oncology surgery. It aims to identify elderly ( $>65$ years old) and frail patients and our discussion in this chapter is relevant to elective liver resection.

\section{CURRENT IMPLEMENTATION OF ROSE}

\section{Target population}

Not all elderly patients are the same and hence patient selection is essential to streamline the resources. One method of patient selection is by virtue of risk prediction. Various risk prediction models are reported in diverse pathologies and surgeries to enhance resource allocation ${ }^{[1,34,35]}$. A prospective study on 162 patients undergoing hepatopancreaticobiliary surgery by van der Windt et al ${ }^{[36]}$, showed that scoring systems such as the Risk Analysis Index, was able to accurately predict post-operative outcomes in patients. The study further elaborates on the possible use of such a scoring system to identify target groups for prehabilitation to optimise outcomes. Tan Tock Seng Hospital Nutrition Screening Tool is a locally developed tool which is validated against subjective global assessment in a cohort of elderly patients ${ }^{[37]}$. In a local study including 281 acute admissions with age range of 61-102 years, Tan Tock Seng Hospital Nutrition Screening Tool predicted risk of malnutrition with high accuracy (area under the curve 0.87) and malnutrition predicted 6-month mortality (adjusted OR $=2.2 ; P=0.05)$ and hospital length of stay ${ }^{[37]}(P<0.05)$.

Currently ROSE program is piloted for patients who undergo liver, pancreas and colorectal surgeries. Initially, patients ( $\geq 65$ years old) are screened for frailty and malnutrition. Those at risk will be enrolled into ROSE program [Figure 2].

\section{Multidisciplinary team}

Upon decision for liver resection, an assessment of frailty and nutrition is done by consultant surgeon. Patient who fulfil the criteria are then sent to allied healthcare members to be enrolled and seen as part of the ROSE program. Members of the program and their role are as follows (further details on workflow in Appendix 1).

\section{Dietician}

Nutritional counseling pre and post operatively: the role of the dietician has been studied in multimodal care teams such as in Poindessous et al. ${ }^{[38]}$ which demonstrates the benefit in a dietician functioning as an educator as well, with decreased recidivism and increased return to independence.

To obtain initial anthropometric and to conduct subjective global assessment: this is especially important in context of liver resection as malnutrition is very common in patients with cirrhosis ${ }^{[39]}$ and to add to this, precise evaluation of their nutrition status is difficult with the presence of ascites and edema ${ }^{[40]}$. A review by Doherty et al ${ }^{[41]}$ identifies intra hepatic fat as an independent risk factor for post-operative morbidity following hepatic resection. Dietary interventions such as calorie restriction, carbohydrate restriction or a Mediterranean diet have shown reductions in pre-operative intra hepatic fat by up to $55 \%{ }^{[40]}$. 


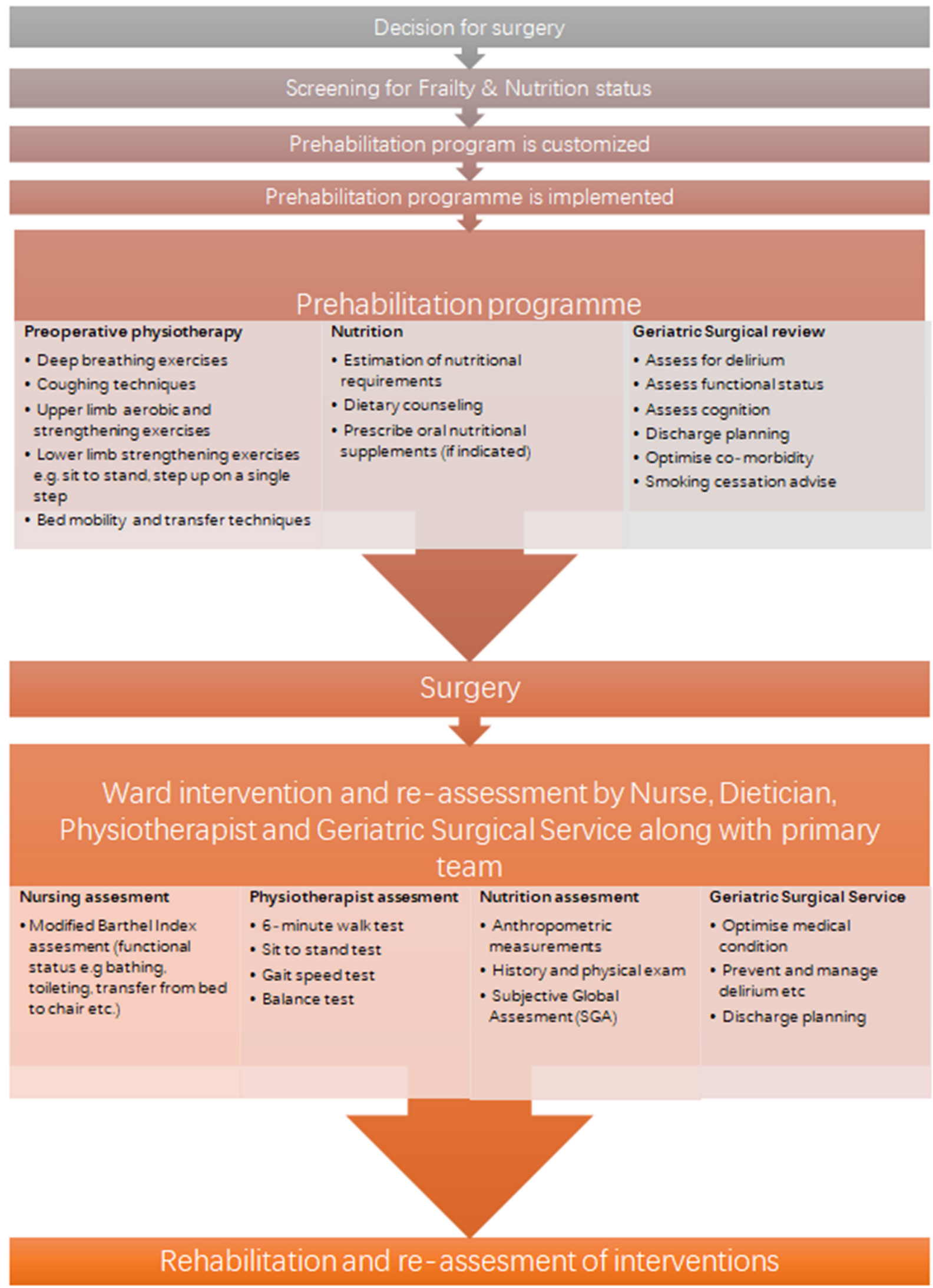

Figure 2. Recovery of surgery in elderly patient journey flowchart 
Nutritional supplementation and monitoring pre and post-operatively: supplementation could include Branch chain amino acids for protein and glycogen synthesis promotion and for the regulation of immune system $^{[40]}$.

Glutamine is a conditionally essential amino acid and is a fuel for neutrophils, lymphocytes and enterocytes $^{[42]}$. A Cochrane review ${ }^{[43]}$ reporting 4671 patients with critical illness or elective major surgery showed that glutamine supplementation reduced infection rates by $21 \%$ and days on mechanical ventilation by 0.69 days.

\section{Physiotherapist}

To assess objective and subjective markers of functional capacity pre and post-operatively: 6-min walk test, sit to stand test, Gait speed test and balance tests. Structured exercise program, either inpatient or outpatient is essential to prehabilitation ${ }^{[4]}$, these show improved outcomes such as in functional physical tests such as the $6 \mathrm{MWT}$.

To conduct physiotherapy sessions pre operatively to increase functional capacity, respiratory function and mobility training in preparation for post-operative period: in a review by Guinan et al. ${ }^{[45]}$ on patients who underwent esophagectomies, it was reported that inspiratory muscle training conducted by physiotherapists may improve post-operative outcomes such as lowering hospitalization by 4.5 days and reducing the rate of high grade post-operative pulmonary complications.

To conduct physiotherapy as required to rehabilitate to baseline fitness post operatively.

\section{Nurse}

Modified Barthel Index assessment pre and post operatively: this is to ascertain patients pre and postoperative baselines and ensure return to function. In a study by Fortinsky et al. ${ }^{[46]}$ on 89 chronically ill patients, the Modified Barthel Index was able to reliably measure and track patient's ability to perform activities of daily living effectively. Furthermore, the Modified Barthel Index was easy to administer without much variability in reporting activities of daily living ${ }^{[47]}$.

\section{Geriatric surgery service}

Peri-operative medicine aims to provide high quality evidence based cross-specialty multidisciplinary care for surgical patients. Partridge et al. ${ }^{[48]}$ has reported a prospective randomized controlled study including 176 patients aged 65 years and older undergoing elective aortic aneurysm repair or lower-limb arterial surgery comparing standard preoperative assessment with preoperative comprehensive geriatric assessment and optimization. They reported lower incidence of delirium ( $11 \%$ vs. $24 \%, P=0.018)$, cardiac complications ( $8 \%$ vs. $27 \%, P=0.001)$ and bladder/bowel complications $(33 \% v s .55 \%, P=0.003)$ in the intervention group. We have a geriatric surgical service capability with a consultant lead team and includes geriatric trained nursing staff. The team assists in managing diverse care needs upon referral: (1) optimize medical co-morbidity and assist with peri-operative care; (2) combined care provided by the geriatrician, anesthesiologist and surgeon is pivotal in the functioning of the prehabilitation program ${ }^{[49]}$.

\section{Financial counselor}

Finance is an important element of health care and often considered less important in context of public healthcare as treatment/intervention is "paid for" by the state in many high income countries. In Singapore, patient co-share the cost burden and hence it is imperative to appropriately counsel patients with estimated bill size and how does it vary based on different choices of therapy, e.g., open surgery vs. laparoscopic surgery, etc. A trained professional supplements the multidisciplinary team for this task and the role is: (1) to ensure financial security and if needed to counsel patients on financial aid programs pre operatively; (2) 
financial counselors aim to allow patients to attain financial security through: inpatient bill estimates to appropriately plan, advice on available financial schemes, subsidies and aid, payment modalities available.

\section{Anesthesiologist}

To conduct pre admission anesthesia risk assessment for patient, these are done through pre-anesthetic clinics in which all pre-operative testing, specialist consultation, nursing and laboratory reviews are integrated into a one-stop clinic with minimal repeat visits. This has also been shown to reduce overall healthcare costs and manpower wastage due to cancellations ${ }^{[50]}$.

\section{Case manager}

To oversee patient journey and ensure seamless process through the ROSE program, e.g., same day appointment scheduling, etc.

In conclusion, age itself is not a risk factor for inferior peri-operative outcomes of elderly patient undergoing liver resection. Co-morbidities should be optimized prior to elective surgery. With an ageing society, prehabilitation has an important and integral role in surgical care. Structured programs involving multidisciplinary teams are essential to enhance peri-operative outcomes. To increase compliance and ensure direct oversight of implementation, resources are needed. Further research into the efficacy of specific prehabilitation regimes is needed to guide patient selection, define best practices and establish the value of such initiatives.

\section{DECLARATIONS}

\section{Authors' contributions}

Conceived of the presented idea: Shelat VG

Wrote the manuscript (under supervision from Shelat VG): Mohan R

Edited and provided critic to the manuscript: Shelat VG

Provided inputs and contributed to final manuscript: Mohan R, Huey CWT, Junnarkar S, Low JK

\section{Availability of data and materials}

Not applicable.

\section{Financial support and sponsorship}

None.

\section{Conflicts of interest}

All authors declared that there are no conflicts of interest.

\section{Ethical approval and consent to participate}

Not applicable.

\section{Consent for publication}

Not applicable.

\section{Copyright}

(c) The Author(s) 2020.

\section{REFERENCES}

1. Ahmed S, de Souza NN, Qiao W, Kasai M, Keem LJ, et al. Quality of life in hepatocellular carcinoma patients treated with transarterial chemoembolization. HPB Surg 2016;2016:6120143. 
2. Boss GR, Seegmiller JE. Age-related physiological changes and their clinical significance. West J Med 1981;135:434-40.

3. Cruz-Jentoft AJ, Baeyens JP, Bauer JM, Boirie Y, Cederholm T, et al.; European Working Group on Sarcopenia in Older People. Sarcopenia: European consensus on definition and diagnosis: report of the European Working Group on Sarcopenia in Older People. Age Ageing 2010;39:412-23.

4. Otsuji H, Yokoyama Y, Ebata T, Igami T, Sugawara G, et al. Preoperative sarcopenia negatively impacts post-operative outcomes following major hepatectomy with extrahepatic bile duct resection. World J Surg 2015;39:1494-500.

5. Hayashi K, Yokoyama Y, Nakajima H, Nagino M, Inoue T, et al. Preoperative 6-minute walk distance accurately predicts post-operative complications after operations for hepato-pancreato-biliary cancer. Surgery 2017;161:525-32.

6. Morley JE. Frailty and sarcopenia in elderly. Wien Klin Wochenschr 2016;128:439-45.

7. Coudeyre E, Jardin C, Givron P, Ribinik P, Revel M, Rannou F. Could preoperative rehabilitation modify post-operative outcomes after total hip and knee arthroplasty? Elaboration of French clinical practice guidelines. Annales de Réadaptation et de Médecine Physique 2007;50:189-97.

8. Rooks DS, Huang J, Bierbaum BE, Bolus SA, Rubano J, et al. Effect of preoperative exercise on measures of functional status in men and women undergoing total hip and knee arthroplasty. Arthritis Rheum 2006;55:700-8.

9. Oosting E, Jans MP, Dronkers JJ, Naber RH, Dronkers-Landman CM, et al. Preoperative home-based physical therapy versus usual care to improve functional health of frail older adults scheduled for elective total hip arthroplasty: a pilot randomized controlled trial. Arch Phys Med Rehabil 2012;93:610-6.

10. Au D, Matthew AG, Lopez P, Hilton WJ, Awasthi R, et al. Prehabilitation and acute postoperative physical activity in patients undergoing radical prostatectomy: a secondary analysis from an RCT. Sports Med Open 2019;5:18.

11. Painter PL, Hector L, Ray K, Lynes L, Dibble S, et al. A randomized trial of exercise training after renal transplantation. Transplantation 2002;74:42-8.

12. Takahashi A, Hu SL, Bostom A. Physical activity in kidney transplant recipients: a review. Am J Kidney Dis 2018;72:433-43.

13. Romano G, Lorenzon E, Montanaro D. Effects of exercise in renal transplant recipients. World J Transplant 2012;2:46-50.

14. Mayo NE, Feldman L, Scott S, Zavorsky G, Kim DJ, et al. Impact of preoperative change in physical function on postoperative recovery: argument supporting prehabilitation for colorectal surgery. Surgery 2011;150:505-14.

15. Gillis C, Buhler K, Bresee L, Carli F, Gramlich L, et al. Effects of nutritional prehabilitation, with and without exercise, on outcomes of patients who undergo colorectal surgery: a systematic review and meta-analysis. Gastroenterology 2018;155:391-410.e4.

16. Cabilan CJ, Hines S, Munday J. The effectiveness of prehabilitation or preoperative exercise for surgical patients: a systematic review. JBI Database System Rev Implement Rep 2015;13:146-87.

17. Rolving N, Nielsen CV, Christensen FB, Holm R, Bünger CE, et al. Does a preoperative cognitive-behavioral intervention affect disability, pain behavior, pain, and return to work the first year after lumbar spinal fusion surgery? Spine 2015;40:593-600.

18. Madhavan S, Shelat VG, Soong SL, Woon WWL, Huey T, et al. Predicting morbidity of liver resection. Langenbecks Arch Surg 2018;403:359-69.

19. Tang EL, Shelat VG, Junnarkar SP, Huey CWT, Low JK, et al. Predictive value of post-operative drain amylase levels for post-operative pancreatic fistula. Ann Hepatobiliary Pancreat Surg 2018;22:397-404.

20. Nakajima H, Yokoyama Y, Inoue T, Nagaya M, Mizuno Y, et al. Clinical benefit of preoperative exercise and nutritional therapy for patients undergoing hepato-pancreato-biliary surgeries for malignancy. Ann Surg Oncol 2019;26:264-72.

21. Seow JG, Lim YR, Shelat VG. Low serum albumin may predict the need for gastric resection in patients with perforated peptic ulcer. Eur J Trauma Emerg Surg 2017;43:293-8.

22. Ausania F, Senra P, Meléndez R, Caballeiro R, Ouviña R, et al. Prehabilitation in patients undergoing pancreaticoduodenectomy: a randomized controlled trial. Rev Esp Enferm Dig 2019;111:603-8.

23. Janssen TL, Steyerberg EW, Langenberg JCM, de Lepper CCHAVH, Wielders D, et al. Multimodal prehabilitation to reduce the incidence of delirium and other adverse events in elderly patients undergoing elective major abdominal surgery: an uncontrolled before-and-after study. PLoS One 2019;14:e218152.

24. Nielsen PR, Jørgensen LD, Dahl B, Pedersen T, Tønnesen H. Prehabilitation and early rehabilitation after spinal surgery: randomized clinical trial. Clin Rehabil 2010;24:137-48.

25. Wang B, Shelat VG, Chow JJL, Huey TCW, Low JK, et al. Prehabilitation program improves outcomes of patients undergoing elective liver resection. J Surg Res 2020;251:119-25.

26. Dunne DF, Jack S, Jones RP, Jones L, Lythgoe DT, et al. Randomized clinical trial of prehabilitation before planned liver resection. Br J Surg 2016;103:504-12.

27. Tandon P, Ismond KP, Riess K, Duarte-Rojo A, Al-Judaibi B, et al. Exercise in cirrhosis: Translating evidence and experience to practice. J Hepatol 2018;69:1164-77.

28. Locklear CT, Golabi P, Gerber L, Younossi ZM. Exercise as an intervention for patients with end-stage liver disease: systematic review. Medicine (Baltimore) 2018;97:e12774.

29. Wimble K, Jones C. Enhanced recovery after surgery for liver resection surgery. Dig Med Res 2019;2. Available from: http://dmr. amegroups.com/article/view/5386 [Last accessed on 20 Mar 2020]

30. Brustia R, Slim K, Scatton O. Enhanced recovery after liver surgery. J Visc Surg 2019;156:127-37.

31. Strong for Surgery. Available from: https://www.facs.org/quality-programs/strong-for-surgery [Last accessed on 20 Mar 2020]

32. Shelat VG, Chia VJ, Low J. Common bile duct exploration in an elderly Asian population. Int Surg 2015;100:261-7.

33. Martínez-Cecilia D, Cipriani F, Shelat V, Ratti F, Tranchart H, et al. Laparoscopic versus open liver resection for colorectal metastases 
in elderly and octogenarian patients: a multicenter propensity score based analysis of short- and long-term outcomes. Ann Surg 2017;265:1192-200.

34. Anbalakan K, Chua D, Pandya GJ, Shelat VG. Five year experience in management of perforated peptic ulcer and validation of common mortality risk prediction models - are existing models sufficient? A retrospective cohort study. Int J Surg 2015;14:38-44.

35. Kiat TTJ, Gunasekaran SK, Junnarkar SP, Low JK, Woon W, et al. Are traditional scoring systems for severity stratification of acute pancreatitis sufficient? Ann Hepatobiliary Pancreat Surg 2018;22:105-15.

36. van der Windt DJ, Bou-Samra P, Dadashzadeh ER, Chen X, Varley PR, et al. Preoperative risk analysis index for frailty predicts shortterm outcomes after hepatopancreatobiliary surgery. HPB (Oxford) 2018;20:1181-8.

37. Lim YP, Lim WS, Tan TL, Daniels L. Evaluating the validity of a nutritional screening tool in hospitalized older adults. Ann Acad of Med Singapore 2008;37:S5.

38. Poindessous JL, Basta M, Renaud A, Vincent M, Héron A. Infirmière et diététicienne: une collaboration efficace en service de rééducation [Nurse and dietitian: effective collaboration in rehabilitation]. Rev Infirm 2018;67:39-41. (in French)

39. Anastácio LR, Davisson Correia MI. Nutrition therapy: integral part of liver transplant care. World J Gastroenterol 2016;22:1513-22.

40. Masuda T, Shirabe K, Yoshiya S, Matono R, Morita K, et al. Nutrition support and infections associated with hepatic resection and liver transplantation in patients with chronic liver disease. JPEN J Parenter Enteral Nutr 2013;37:318-26.

41. Doherty DT, Coe PO, Rimmer L, Lapsia S, Krige A, et al. Hepatic steatosis in patients undergoing resection of colorectal liver metastases: a target for prehabilitation? A narrative review. Surg Oncol 2019;30:147-58.

42. Shelat VG, Pandya GJ. Nutritional Support in dysphagia. Available from: https://www.intechopen.com/books/seminars-in-dysphagia/ nutritional-support-in-dysphagia [Last accessed on 20 Mar 2020]

43. Tao KM, Li XQ, Yang LQ, Yu WF, Lu ZJ, et al. Glutamine supplementation for critically ill adults. Cochrane Database Syst Rev 2014;2014:CD010050.

44. Carli F, Scheede-Bergdahl C. Prehabilitation to enhance peri-operative care. Anesthesiol Clin 2015;33:17-33.

45. Guinan EM, Dowds J, Donohoe C, Reynolds JV, Hussey J. The physiotherapist and the esophageal cancer patient: from prehabilitation to rehabilitation. Dis Esophagus 2017;30:1-12.

46. Fortinsky RH, Granger CV, Seltzer GB. The use of functional assessment in understanding home care needs. Med Care 1981;19:489-97.

47. Collin C, Wade DT, Davies S, Horne V. The Barthel ADL Index: a reliability study. Int Disabil Stud 1988;10:61-3.

48. Partridge JS, Harari D, Martin FC, Peacock JL, Bell R, et al. Randomized clinical trial of comprehensive geriatric assessment and optimization in vascular surgery. Br J Surg 2017;104:679-87.

49. Carli F, Ferreira V. Prehabilitation: a new area of integration between geriatricians, anesthesiologists, and exercise therapists. Aging Clin Exp Res 2018;30:241-4.

50. Gupta A, Gupta N. Setting up and functioning of a preanaesthetic clinic. Indian J Anaesth 2010;54:504-7. 